\title{
Epidémiologie, Diagnostic et Prise en Charge des Tumeurs Primitives de L'appendice Au Centre Hospitalier Universitaire Sylvanus Olympio (CHU SO) de Lomé Au Togo
}

\author{
Kodjo Abossisso Sakiye, \\ Eyram Sorsy, \\ Fousséni Alassani, \\ Kokou Kanassoua, \\ Badjona Songne- Gnamkoulamba
}

Département de Chirurgie et Spécialités Chirurgicales, Service des Urgences Chirurgicales, CHU Sylvanus Olympio, Faculté des Sciences de la Santé, Université de Lomé, Togo

\section{Résumé}

Objectif : préciser les circonstances de découvertes des tumeurs primitives de l'appendice et apprécier les résultats de la prise en charge dans nos conditions d'exercice de travail au CHU SO de Lomé au Togo. Méthodologie : étude rétrospective descriptive incluant les dossiers des patients pris en charge dans le service des urgences du CHU SO pour appendicite aigue et chez qui l'examen anatomo-pathologique de la pièce d'appendicectomie avait confirmé une tumeur primitive de l'appendice, de Janvier 2014 à Décembre 2018 (5 ans).Résultats : nous rapportons 5 cas de tumeurs appendiculaires découvertes chez 3 femmes et 2 hommes. L'âge moyen des patients était de 32,8 ans. La symptomatologie clinique était celle d'une appendicite aigue confirmée à la tomodensitométrie (TDM). L'indication chirurgicale avait été posée et le geste chirurgical avait consisté à une appendicectomie dans tous les cas. L'examen histologique avait permis de découvrir fortuitement une tumeur primitive de l'appendice dont 4 tumeurs neuroendocrines et un adénocarcinome. Une hémicolectomie complémentaire avait été réalisée dans le cas de l'adénocarcinome. Conclusion : les tumeurs primitives de l'appendice sont rares et leur expression symptomatique est celle d'un tableau d'une appendicite aigue. Seul l'examen anatomopathologique de la pièce d'appendicectomie permet de diagnostiquer fortuitement une tumeur primitive de l'appendice. 
Mots clés : Appendice, Tumeur, Primitive

\title{
Epidemiology, Diagnosis and Management of Primary Tumors of The Appendix at The Sylvanus Olympio University Hospital (CHU SO) in Lomé, Togo
}

\author{
Kodjo Abossisso Sakiye, \\ Eyram Sorsy, \\ Fousséni Alassani, \\ Kokou Kanassoua, \\ Badjona Songne- Gnamkoulamba \\ Département de Chirurgie et Spécialités Chirurgicales, \\ Service des Urgences Chirurgicales, CHU Sylvanus Olympio, \\ Faculté des Sciences de la Santé, Université de Lomé, Togo
}

\begin{abstract}
Background: to clarify the circumstances of discovery of the primary tumors of the appendix and to assess the results of management in our working conditions at the University Hospital Sylvanus Olympio Lomé in Togo. Methodology: retrospective descriptive study including records of patients treated in the emergency department of the Sylvanus Olympio University Hospital Center for acute appendicitis and in whom the pathological examination of the appendectomy specimen confirms a primary tumor of the appendix, from January 2014 to December 2018 (5 years). Results: we report 5 cases of appendicular tumors found in 3 women and 2 men. The average age of the patients was 32.8 years old. The clinical symptomatology was that of acute appendicitis confirmed by computed tomography (CT). The surgical indication was asked and the surgical procedure consisted of an appendectomy in all cases. Histological examination revealed accidentally a primary appendix tumor including 4 neuroendocrine tumors and adenocarcinoma. A complementary hemi colectomy was performed in the case of adenocarcinoma. Conclusion: the primary tumors of the appendix are rare and their symptomatic expression is that of a table of acute appendicitis. Only the anatomopathological examination of the appendectomy specimen can accidentally diagnose a primary tumor of the appendix.
\end{abstract}

Keywords: Appendix, Tumor, Primitive 


\section{Introduction}

Les tumeurs primitives de l'appendice sont rares et d'évolution lente. Elles sont dominées par les tumeurs neuroendocrines (Khan, 2019 ; Misdraji, 2005). Découverte de façon fortuitement sur l'histologie après une appendicectomie pour une appendicite aiguë, leur prévalence est de 0,3 à $0,9 \%$ chez les patients appendicectomisés. L'examen histologique permet de les identifier dans 0,1 à $0,5 \%$ des pièces d'appendicectomie (Khan, 2019 ; Misdraji, 2005 ; Khabir, 2000 ; Connor, 1998 ; Deans, 1995). Leur symptomatologie est non spécifique et est dominé par un syndrome appendiculaire ou un tableau d'appendicite aigue. Leur diagnostic préopératoire est exceptionnel malgré l'apport de l'imagerie et repose encore sur l'anatomopathologique dans la plupart des cas (Khan, 2019 ; Misdraji, 2005 ; Khabir, 2000 ; Connor, 1998 ; Deans, 1995). La prise en charge des syndromes appendiculaires reste dominée par une simple appendicectomie du fait du tableau clinique d'une appendicite aigue et le pronostic est fonction du type histologique, de la taille de la tumeur et du degré d'infiltration pariétale (Khabir, 2000 ; Connor, 1998). Par conséquent, de nombreuses tumeurs appendiculaires malignes sont diagnostiquées à un stade avancé, ce qui entraîne un pronostic défavorable par rapport aux cancers du côlon habituels (Lee, 2011 ; Ko, 2010).

$\mathrm{Au}$ Togo, les tumeurs digestives sont de plus en plus prise en charge et étudiées mais très peu d'études sont consacrées aux tumeurs appendiculaires. De plus, les moyens financiers limités des patients et le caractère banal des appendicites aigues font que les pièces opératoires ne font pas systématiquement l'objet d'un examen histologique ; ce qui constitue une erreur grave d'autant plus que la pathologie appendiculaire peut cacher une tumeur primitive de l'appendice. Nous rapportons dans notre étude 5 cas dans le but de préciser les circonstances de découvertes et d'apprécier les résultats de la prise en charge dans nos conditions d'exercice de travail dans le service des urgences chirurgicales du CHU SO de Lomé au Togo.

\section{Patients et méthodes}

Il s'agissait d'une étude rétrospective descriptive incluant les dossiers des patients pris en charge dans le service des urgences du CHU SO pour appendicite aigue et chez qui l'examen anatomo-pathologique de la pièce d'appendicectomie avait confirmé une tumeur primitive de l'appendice. Cette étude portait sur une période de 05 ans allant de Janvier 2014 à Décembre 2018. Elle a intéressé 05 patients dont 03 de sexe féminin et 02 de sexe masculin. 


\section{Résultats}

Cette étude rapporte 05 cas de tumeurs primitives de l'appendice confirmées à l'examen anatomopathologique. L'âge des patients était de 19, 21, 55, 61 et 82 ans, soit une moyenne de 32,8 ans. Il y avait 3 femmes pour 2 hommes.

La symptomatologie évoluait depuis moins de 24 heures dans 2 cas. Dans les autres cas, elle durait depuis plus de 24 heures. Dans 2 cas, les douleurs abdominales évoluaient de façon paroxystique depuis 2 semaines. Cliniquement, elle était faite de douleurs abdominales isolées localisées en fosse iliaque droite (FID) d'apparition brutale dans 3 cas, associée à des nausées et vomissements dans 1 cas. Dans tous les cas, Il n'y avait pas de fièvre avec une température moyenne de $37,1^{\circ} \mathrm{C}\left(36,9^{\circ} \mathrm{C}-37,3^{\circ} \mathrm{C}\right)$. Il n'y avait pas de troubles du transit dans tous les cas. Par contre, il existait une notion d'amaigrissement avec une perte de poids estimé à $10 \mathrm{~kg}$ en 3 mois dans 1 cas. L'examen clinique mettait en évidence dans 3 cas une défense localisée en FID au point de Mac Burney et dans 2 cas des douleurs provoquées en FID à la décompression brutale de la paroi abdominale (signe de Blumberg). Le tableau 1 présente la répartition des différents éléments cliniques. Les résultats du bilan biologique et du scanner sont résumés dans le tableau 2.

Tableau 1 : répartition des différents éléments cliniques par patient

\begin{tabular}{|l|l|l|l|l|l|}
\hline & $\begin{array}{l}\text { S.G. } \\
\text { M19ans }\end{array}$ & $\begin{array}{l}\text { N. M. } \\
\text { F21ans }\end{array}$ & $\begin{array}{l}\text { P. O. } \\
\text { F55ans }\end{array}$ & $\begin{array}{l}\text { V. M. } \\
\text { F61ans }\end{array}$ & $\begin{array}{l}\text { D. D. } \\
\text { M82ans }\end{array}$ \\
\hline $\begin{array}{l}\text { Douleurs localisées } \\
\text { en FID }\end{array}$ & + & + & + & + & + \\
\hline Début & brutal & Brutal & progressif & brutal & progressif \\
\hline Température $\left({ }^{\circ} \mathrm{C}\right)$ & 36.9 & 37.2 & 37 & 37.2 & 37.3 \\
\hline $\begin{array}{l}\text { Nausées } \\
\text { vomissements }+/-\end{array}$ & oui & 0 & 0 & 0 & 0 \\
\hline Amaigrissement & non & Non & non & non & oui \\
\hline diarrhées & & & 0 & 0 & 0 \\
\hline
\end{tabular}

$\mathrm{M}=$ masculin, $\mathrm{F}=$ féminn.

Tableau 2 : Récapitulatif des résultats du bilan biologique et du scanner.

\begin{tabular}{|l|l|l|l|}
\hline & GB & CRP & Résultats du Scanner \\
\hline D. D. & 7600 & 50 & $\begin{array}{l}\text { Abcès appendiculaire avec collection } \\
\text { dans le cul-de-sac de Douglas }\end{array}$ \\
\hline V. M. & 11200 & 97,3 & Mucocèle appendiculaire rétro-coecal, \\
\hline N. M. & 16400 & 25,6 & Appendicite aigue non compliquée. \\
\hline S. G. & 13900 & 55,2 & $\begin{array}{l}\text { Aspect scanographique d'une appendicite } \\
\text { aigue stercorale sans signe } \\
\text { scanographique de complication. } \\
\text { Épanchement intra péritonéal minime } \\
\text { dans le cul-de-sac de Douglas. }\end{array}$ \\
\hline P. O. & 700 & 1,5 & \begin{tabular}{l} 
Mucocèle appendiculaire invaginé \\
\hline
\end{tabular} \\
\hline
\end{tabular}


L'indication chirurgicale avait été posée dans tous les cas et le geste consistait en une appendicectomie avec comme consigne une gestion prudente de l'appendice surtout dans les 2 cas de mucocèle en vue d'éviter toute rupture dans la cavité péritonéale. Au cours de l'opération, une lésion suspecte de l'appendice avait été portée dans 2 cas seulement. La voie d'abord, l'aspect macroscopique et les résultats de l'examen anatomo-pathologique sont présentés dans le tableau 3.

Tableau 3 : Voies d'abords, aspects macroscopiques de l'appendice et résultats de l'examen anatomo-pathologique des pièces d'appendicectomie.

\begin{tabular}{|c|c|c|c|}
\hline & Voie d'abord & $\begin{array}{l}\text { Aspect macroscopique de } \\
\text { l'appendice }\end{array}$ & $\begin{array}{l}\text { Résultats de l'examen anatomo- } \\
\text { pathologique des pièces } \\
\text { d'appendicectomie }\end{array}$ \\
\hline D. D. & cœlioscopie & $\begin{array}{l}\text { PLASTRON } \\
\text { APPENDICULAIRE : A } \\
\text { l'extrémité de l'appendice } \\
\text { on observe une } \\
\text { volumineuse lésion } \\
\text { blanchâtre un peu colloïde } \\
\text { développée sur le bord } \\
\text { anti-méso-appendiculaire } \\
\text { et qui atteint la marge } \\
\text { circonférentielle. }\end{array}$ & $\begin{array}{l}\text { Présence au niveau de la pointe } \\
\text { appendiculaire, d'un } \\
\text { adénocarcinome moyennement } \\
\text { différencié Muci sécrétant } \\
\text { infiltrant le méso-appendice. La } \\
\text { tumeur atteint la marge } \\
\text { circonférentielle. Un nodule } \\
\text { centimétrique remis à part } \\
\text { correspond également à } \\
\text { l'adénocarcinome. La recoupe } \\
\text { chirurgicale colique est saine. }\end{array}$ \\
\hline V. M. & cœlioscopie & $\begin{array}{l}\text { Appendice très } \\
\text { volumineux typique d'un } \\
\text { mucocèle }\end{array}$ & $\begin{array}{l}\text { Mucocèle appendiculaire non } \\
\text { rompu et sans envahissement de la } \\
\text { paroi ; il s'accompagne d'un } \\
\text { Cystadénome mucineux } \\
\text { appendiculaire de bas grade, } \\
\text { occupant moins du tiers de la } \\
\text { circonférence, située à proximité } \\
\text { de la pointe appendiculaire et à } \\
\text { plus de } 4 \mathrm{~cm} \text { de la limite d'exérèse }\end{array}$ \\
\hline N. M. & cœlioscopie & $\begin{array}{l}\text { Appendice phlegmoneux } \\
\text { avec de fausses } \\
\text { membranes et infiltration } \\
\text { de la graisse au contact }\end{array}$ & $\begin{array}{l}\text { Appendicite aigue suppurée avec } \\
\text { tumeur carcinoïde de la pointe } \\
\text { appendiculaire de } 1 \mathrm{~mm}\end{array}$ \\
\hline S. G. & cœlioscopie & $\begin{array}{l}\text { Appendice phlegmoneux } \\
\text { avec des adhérences épipo- } \\
\text { digestives }\end{array}$ & $\begin{array}{l}\text { Appendicite aigue phlegmoneuse. } \\
\text { Découverte au niveau de la pointe } \\
\text { appendiculaire d'une tumeur } \\
\text { neuroendocrine de } 9 \mathrm{~mm} \text { de grand } \\
\text { axe infiltrant localement la sous- } \\
\text { séreuse mais respectant le } \\
\text { revêtement péritonéal bien } \\
\text { différenciée et dont l'index de } \\
\text { prolifération est bas égal à } 1 \% \\
\text { (pT3G1). Recoupe proximale } \\
\text { saine. }\end{array}$ \\
\hline
\end{tabular}




\begin{tabular}{|l|l|l|l|}
\hline P. O. & $\begin{array}{l}\text { Celioscopie exploratrice } \\
\text { +laparotomie }\end{array}$ & $\begin{array}{l}\text { Petite invagination iléo- } \\
\text { caecale avec un appendice } \\
\text { sous tension : ouverture du } \\
\text { bas fond caecal avec } \\
\text { résection de toute la base } \\
\text { d'implantation de } \\
\text { l'appendice }\end{array}$ & $\begin{array}{l}\text { Mucocèle appendiculaire avec } \\
\text { dysplasie glandulaire de bas grade } \\
\text { au niveau de la muqueuse. } \\
\text { Absence de prolifération } \\
\text { adénocarcinomateuse sur } \\
\text { l'ensemble du matériel examiné. }\end{array}$ \\
\hline
\end{tabular}

Les suites opératoires immédiates avaient été simples dans tous les cas. Secondairement, une éventration avait été découverte dans 1 cas, notamment chez la patiente qui a bénéficié d'une cœlioscopie exploratrice suivie d'une laparotomie complémentaire.

Dans un cas, il avait été découvert la présence au niveau de la pointe appendiculaire d'un adénocarcinome moyennement différencié muci sécrétant, infiltrant le méso-appendice avec une marge circonférentielle atteinte et un nodule centimétrique remis à part correspond également à l'adénocarcinome. Une discussion collégiale avait décidé de la réalisation d'une chirurgie complémentaire qui avait consisté en une hémicolectomie droite carcinologique. Les suites opératoires avaient été simples et l'examen anatomo-pathologique de la pièce opératoire était indemne de lésion néoplasique (19 ganglions mésentériques sains).

\section{Discussion}

Les tumeurs malignes développées primitivement aux dépens des tissus de l'appendice sont rares et sont dominées par des tumeurs neuroendocrines (Khan, 2019 ; Misdraji, 2005). Elles représentaient 0,57\% des pièces d'appendicectomie dans notre série avec $4 / 5$ de tumeurs carcinoïdes appendiculaires. L'âge moyen des patients était de 32,8ans avec une prédominance féminine. Khan et collaborateurs (Khan, 2019) ont aussi trouvé un âge moyen des patients de 31,73 ans, avec $81,8 \%$ de femmes; ces faits ont été aussi rapportés par Khabir et collaborateurs (Khabir, 2000), Edmonds et collaborateurs (Edmonds, 1984) et Butler et collaborateurs (Butler, 1994). Ces tumeurs sont localisées dans 70 à $84 \%$ au niveau de la pointe appendiculaire ; leur taille dépasse $1 \mathrm{~cm}$ dans $20 \%$ des cas $[4,5]$; dans notre série, elles étaient toutes situées à la pointe de l'appendice et leur taille était inférieure à $1 \mathrm{~cm}$ dans la moitié des cas. Pour Khabir et collaborateurs (Khabir, 2000), la taille est inférieure à $1 \mathrm{~cm}$ dans $90 \%$; cette différence de taille s'explique probablement par la taille des échantillons.

Les adénocarcinomes appendiculaires sont plus rares et retrouvés dans 0,01 à $0,11 \%$ des pièces d'appendicectomie (Lee, 2011; Benedix, 2010, Limaiem, 2015 ; Oya, 2009). Il y avait 1cas dans notre série. Selon Lenriot et collaborateurs (Lenriot, 1988), ils surviennent souvent sur un adénome préexistant avec un âge moyen de 50 ans et une prédominance masculine ; 
notre seul cas d'adénocarcinome était de sexe féminin âgé de 63 ans (un âge supérieur à celui de Lenriot et collaborateurs (Lenriot, 1988) mais comparable à celui de Khabir et collaborateurs(Khabir, 2000). Cette différence de sexe s'expliquerait par la taille de notre échantillon.

L'association synchrone ou métachrone d'un adénocarcinome appendiculaire à un carcinome colorectal est noté dans $11 \%$ des cas (Karr, 1995). Aucun autre cancer d'un autre organe n'était associé à la tumeur appendiculaire découvert dans notre série.

La symptomatologie clinique des tumeurs primitives de l'appendice est non spécifique et caractérisée par un syndrome appendiculaire et/ou un tableau d'appendicite aigue avec une masse en FID (Khabir, 2000 ; Connor, 1998 ; Lenriot, 1988). Dans notre série la symptomatologie clinique était celle d'une appendicite aigue caractérisée par des douleurs abdominales localisées en fosse iliaque droite associées à des nausées et/ou vomissements sans troubles du transit et le bilan biologique notait une hyperleucocytose dans 3 cas. Le scanner abdominal avec injection avait permis de conclure à une appendicite aigue dans 3 cas et à une mucocèle appendiculaire dans 2 cas. De plus dans notre série, l'expression radiologique du cas d'adénocarcinome rapportait des images scannographiques d'un abcès appendiculaire avec collection dans le cul-de-sac de Douglas; et seul l'examen histologique des pièces d'appendicectomie avait permis de confirmer de l'origine tumorale des symptômes. Dans la série de Khan et collaborateurs (Khan, 2019), la symptomatologie présentée par tous les patients permettait de conclure à une appendicite aigue présumée et seule l'histologie avait permis de découvrir les néoplasies appendiculaires neuroendocrines. Il en ressort qu'aucune symptomatologie clinique et/ou aucun moyen d'exploration paraclinique lié à l'appendice n'est spécifiquement l'expression d'une tumeur primitive de l'appendice et seul l'examen anatomo-pathologique des pièces d'appendicectomie permet de les découvrir; il y a donc lieux de rendre systématique l'examen histologique de toute pièce d'appendicectomie. Dans 46 à $62 \%$ des cas, il y a une perforation de l'adénocarcinome (Lenriot, 1988) et il existe aussi des pseudotumeurs appendiculaires. Dans notre série, il n'y avait pas de perforation dans le cas d'adénocarcinome et aucune pseudotumeur appendiculaire n'avait été observée. Sur le plan thérapeutique, le traitement des tumeurs primitives de l'appendice est chirurgical et autrefois pour la majorité des auteurs il consiste en une simple appendicectomie (Benedix, 2010 ; Parkes, 1993 ; Oya, 2009). De nos jours, la procédure chirurgicale standard pour l'adénocarcinome appendiculaire est l'hémicolectomie droite avec dissection appropriée des ganglions lymphatiques, comme pour les cancers colorectaux courants. L'appendicectomie est considérée comme une procédure optimale et suffisante uniquement dans les lésions confinées à la muqueuse avec une marge 
chirurgicale négative. L'hémicolectomie droite réduit significativement les récidives postopératoires par rapport à une appendicectomie seule. Le taux de survie à 5 ans passe de $20 \%$ après une appendicectomie seule et à $63 \%$ après une hémicolectomie droite (Noriaki, 2012). Ce fut le cas dans notre série où le geste chirurgical avait consisté en une appendicectomie dont 4 par voie coelioscopique et 1 cas par laparotomie transversale sous-ombilicale avec une résection du bas-fond caecal associé à l'appendicectomie.

Le pronostic est fonction du type histologique, de la taille de la tumeur et du degré d'infiltration pariétale; pour les tumeurs carcinoïdes de moins de $2 \mathrm{~cm}$, la survie à 5 ans atteint 100\% (Bourgeon, 1995 ; Deans, 1995). Pour les adénocarcinomes, le pronostic est essentiellement déterminé par le stade de Dukes; la survie à 5 ans passe de $100 \%$ pour le stade A à $6 \%$ pour le stade $\mathrm{D}$ (Deans, 1995). Une hémi colectomie droite serait indiquée dans les tumeurs carcinoïdes supérieures à $2 \mathrm{~cm}$ ou infiltrant la base de l'appendice et dans les adénocarcinomes (Khabir, 2000; Connor, 1998). Ce fut le cas de l'adénocarcinome de notre série ou une hémicolectmie droite carcinologique complémentaire avait été réalisée 1 mois après l'appendicectomie initiale en l'absence d'envahissement ganglionnaire ou de processus évolutif sur le colon; l'examen histologique de la pièce montrait une absence d'envahissement ganglionnaire (19N-) ou de cellule suspecte, et une surveillance avait été instituée.

\section{Conclusion}

Les tumeurs primitives de l'appendice sont rares et sont dominées par des tumeurs neuroendocrines. Leur expression symptomatique est celle d'un tableau d'une appendicite aigue et aucun examen par aclinique surtout d'imagerie médicale ne nous permet de poser le diagnostic préopératoire. Seul l'examen anatomopathologique de la pièce d'appendicectomie permet de diagnostiquer fortuitement une tumeur primitive de l'appendice. Leur traitement reste chirurgical et se résume à une appendicectomie mais des gestes complémentaires peuvent s'avérer utiles telle qu'une hémi colectomie droite carcinologique.

\section{References :}

1. Khan K, Patil S, Roomi S, Shiwani MH.Appendicular Neuroendocrine Neoplasm is Associated with Acute Appendicitis - Don't Miss the Boat. Chirurgia (Bucur). 2019 ; 114(4):461-6.

2. Misdraji J. Neuroendocrine tumours of the appendix. Curr Diag Pathol, 2005, 11, 180-93.

3. Khabir A, Gouiaa N, Boudawara T, Sellami A, Makni S, Beyrouti I, Jlidi R. Tumeurs malignes primitives de l'appendice. Ann Chir 2000; 125: 801-2. 
4. Connor SJ, Hanna GB, Frizelle F.A. Appendiceal tumors: retrospective clinicopathologic analasys of appendiceal tumors for 7.970 appendicectomies. Dis Colon Rectum, 1998, 41, 75-80.

5. Deans G. T. (Deans G.T., Spence R.A.J. Neoplastic lesions of the appendix. Br J Surg 1995; 82 : 299-306.

6. Lee WS, Choi ST, Lee JN, Kim KK, Park YH, Baek JH. Une analyse clinico-pathologique rétrospective de tumeurs appendiculaires à partir de 3744 appendicectomies: une étude monocentrique. Int J Colorectal Dis. 2011; 26: 617-21.

7. Ko YH, parc SH, Jung CK, HS gagné, SH Hong, parc JC, SY Roh, Woo IS, Kang JH, Hong YS, Byun JH. Caractéristiques cliniques et facteurs pronostiques du carcinome appendiculaire primaire. AsiaPac J Clin Oncol. 2010; 6: 19-27.

8. Edmonds P, Merino M, Livolsi V. A, Duray P.H. Adenocarcinoïd (mucinous carcinoïd) of the appendix. Gastroenterology 1984; 86:3029.

9. Butler J. A, Housihar A, Lin F, Wilson S. Goblet cell carcinoïd of the appendix. Am J Surg 1994;168: 685-7.

10. Benedix F, Reimer A, Gastinger I, Mroczkowski P, H Lippert, Kube R. Carcinome appendiculaire primaire - épidémiologie, chirurgie et survie: résultats d'une étude multicentrique allemande. Eur J Surg Oncol. 2010; 36: 763-71.

11. Limaiem F, Arfa N, Marsaoui L, Bouraoui S, Lahmar A, Mzabi S.Unexpected Histopathological Findings in Appendectomy Specimens: a Retrospective Study of 1627 Cases.Indian J Surg. 2015;77(3):1285-90.

12. Oya S, Miyata K, Yuasa N, E Takeuchi, Goto Y, Miyake H, Nagasawa $\mathrm{K}$, Kobayashi Y, Ito T, Ito M. Carcinome précoce de l'appendice vermiformis. Dig Endosc. 2009; 21: 53-5.

13. Lenriot P, Huguier M. Adenocarcinoma of the appendix. Am J Surg 1988; 155:470-5.

14. Karr N. J, McCarthy W, Sobin L. H. Epithelial non carcinoid tumors and tumor-like lesions of the appendix. Cancer 1995; 75:757-68.

15. Parkes S. E., Muir K. R., Alsheyyab M., Cameron A. H., Pincott J. R., Raafat F., and al. Carcinoid tumours of the appendix in children 19571986: incidence, treatment and outcome. Br J Surg 1993; 80:502-4.

16. Oya S, Miyata K, Yuasa N, E Takeuchi, Goto Y, Miyake H, Nagasawa $\mathrm{K}$, Kobayashi Y, Ito T, Ito M. Carcinome précoce de l'appendice vermiformis. Dig Endosc. 2009; 21: 53-5.

17. Noriaki K, Yasutoshi M, Yoshiaki K, Masayoshi N, Kazuma O, Yukihito K, Eigo O. Incidentally Discovered Adenocarcinoma in situ 
of the Appendix in a Young Woman. Case Rep Gastroenterol. 2012 Sep-Dec; 6(3): 726-33.

18. Bourgeon A, Baque P, Valla J, Fabiani P. Conduite à tenir devant une tumeur carcinoïde de l'appendice. Ann Gastroentérol Hépatol 1995 ; $31: 134-7$.

19. Deans G. T. (Deans G.T., Spence R.A.J. Neoplastic lesions of the appendix. Br J Surg $1995 ; 82$ : 299-306). 